\title{
Therapies to Increase ApoA-I and HDL-Cholesterol Levels
}

\author{
William M. Brown and Fabrizio S. Chiacchia \\ Resverlogix Corp., 202, 279 Midpark Way SE, Calgary, AB T2X 1M2, Canada.
}

\begin{abstract}
Cholesterol is transported around the body in the form of lipoprotein (lipid/protein) complexes, because it is almost insoluble in water. High-density lipoprotein (HDL) particles transport cholesterol from tissues back to the liver for excretion. Epidemiological studies have shown an inverse relationship between blood levels of HDL-cholesterol (HDL-c) and the incidence of clinically significant atherosclerosis. The beneficial effects of HDL in altering atherosclerotic disease are believed to involve elevated levels of HDL enhancing the efflux of cholesterol from arterial walls, increasing transport of cholesterol from arteries to the liver for excretion. This reverse cholesterol transport (RCT) pathway is used to explain both HDL's role in lipid metabolism and the inverse association between HDL-c plasma concentration and the risk of cardiovascular disease. Based on the RCT model, ApoA-I is an attractive target for therapeutic intervention. Experimental manipulations to increase production of ApoA-I have been associated with reduced atherogenicity. There is a continuing need for novel therapies that increase the biosynthesis of HDL, to inhibit the progression of and even bring about regression of atherosclerosis. Small molecule compounds that increase the production of endogenous ApoA-I would be attractive therapeutic agents for treating dyslipidemias.
\end{abstract}

Keywords: cholesterol, apolipoprotein A-I (ApoA-I), high-density lipoprotein (HDL), transcription, reverse cholesterol transport (RCT), atherosclerosis, dyslipidemia

\section{Introduction}

Because cholesterol is almost insoluble in water, it is transported around the body in the form of lipoprotein (lipid/protein) complexes. The liver produces very low density lipoproteins (VLDL) and secretes them into plasma, where they are converted to low-density lipoprotein (LDL) particles and non-esterified fatty acids (Schaefer et al. 1978).

High-density lipoprotein (HDL) particles transport cholesterol from tissues back to the liver for excretion, as fecal sterols and bile acids. HDL exists primarily in two forms, one containing apolipoprotein A-I (ApoA-I) and apolipoprotein A-II (ApoA-II), and one containing ApoA-I alone (Schultz et al. 1993). The cardioprotective effect is largely due to ApoA-I. In addition to their role in RCT, HDL particles have anti-inflammatory, anti-oxidative, anti-apoptotic, anti-thrombotic, vasodilatory, and antiinfective properties.

The lipid hypothesis, proposed more than a century ago, is based on the idea that dyslipidemia is central to atherosclerosis (Steinberg 2004, 2005a, 2005b, 2006a, 2006b). The validity of the hypothesis was ultimately established by major epidemiological studies, such as the "Seven Countries Study," which, with a 25-year follow-up, found that across cultures, cholesterol levels were linearly related to coronary heart disease mortality (Verschuren et al. 1995).

Other epidemiological studies, including the Framingham (Gordon et al. 1977) and Procam (Assmann et al. 1982) studies, have shown an inverse relationship between blood levels of HDL-c and the incidence of clinically significant atherosclerosis (Boden, 2000). This inverse association between HDL-c concentrations and cardiovascular risk is apparently continuous; there seems to be no threshold value. In fact, each $1 \mathrm{mg} / \mathrm{dL}$ increment in serum HDL-c is associated with a $2 \%-3 \%$ decrement in cardiovascular risk, while a $1 \%$ reduction in LDL-c decreased the risk of coronary heart disease (CHD) by $1 \%-2 \%$ (Boden, 2000).

The Veterans Affairs HDL-c Intervention Trial (VA-HIT) examined the benefit of secondary prevention in patients with low HDL-c levels and a history of coronary artery disease (CAD). In patients with existing CAD, the only lipid abnormality was a low HDL-c level. In the 2,531 men involved, the average baseline HDL was $32 \mathrm{mg} / \mathrm{dL}$, LDL was $111 \mathrm{mg} / \mathrm{dL}$, and triglycerides (TGs) were $160 \mathrm{mg} / \mathrm{dL}$. Patients received gemfibrozil $(1200 \mathrm{mg} / \mathrm{d})$ or placebo and were followed for 5 years; in those receiving gemfibrozil, HDL increased by $6 \%$ and triglycerides decreased by $31 \%$, with no significant change in

Correspondence: William M. Brown, Ph.D., J.D., Resverlogix Corp., 202, 279 Midpark Way SE, Calgary, AB T2X 1M2, Canada. Tel: 403-254-9252; Fax: 403-256-8495; Email: william@resverlogix.com

Copyright in this article, its metadata, and any supplementary data is held by its author or authors. It is published under the Creative Commons Attribution By licence. For further information go to: http://creativecommons.org/licenses/by/3.0/. 
LDL-c, versus the placebo. Coronary events were reduced by $22 \%$ in the gemfibrozil group (Robins et al. 2001).

The Air Force/Texas Coronary Atherosclerosis Prevention Study demonstrated an association between HDL-c levels and primary prevention of CAD. Participants (men aged 45-73 and postmenopausal women aged 55-73) had normal levels of total and LDL-c, but low HDL-c; they received lovastatin (20-40 mg daily) or placebo. Lovastatin increased HDL by $6 \%$, reduced LDL by $25 \%$, and reduced cardiac events by $37 \%$, versus placebo (Downs et al. 1998).

The Swedish prospective population study, AMORIS, followed 175,000 men and women for 6 years and demonstrated ApoA-I to be the most potent protective factor against fatal myocardial infarction (Walldius et al. 2001). The INTERHEART study, an international case control study comparing myocardial infarction survivors with age- and gender-matched controls, showed the ApoA-I/ApoB ratio to be the strongest modifiable protective factor (Yusuf et al. 2004).

\section{Reverse Cholesterol Transport (RCT)}

While the mechanisms of the beneficial effects of HDL in altering atherosclerotic disease are not completely understood, it is believed that elevated levels of HDL enhance the efflux of cholesterol from arterial walls, increasing transport of cholesterol from arteries to the liver for excretion. Increased ApoA-I increases paraoxonase activity, and enhances anticoagulant and anti-inflammatory activities. HDL also promotes fibrinolysis. Additionally, HDL particles protect against LDL oxidation, a key step in promoting cholesterol uptake by arterial macrophages.

The major steps in the reverse cholesterol transport (RCT) pathway are the active efflux of cholesterol and phospholipids from cells by ATP-binding cassette transporter A1 (ABCA1), the binding of cholesterol to apolipoproteins, forming pre- $\beta$ HDL, the esterification of HDL-bound cholesterol by lecithin cholesterol acyl transferase (LCAT; the resulting cholesteryl esters (CEs) are the core lipids of HDL), CETP-mediated exchange of CEs and TGs between HDL and Apo B-containing particles, and hepatic lipase (HL)-mediated uptake of cholesterol and TGs by the liver (von Eckardstein and Assmann, 1998; see Fig. 1). This RCT model is used to explain both HDL's role in lipid metabolism and the inverse association between HDL-c plasma concentrations and cardiovascular disease risk. The effects of mutations in the various proteins and enzymes of the RCT pathway have helped in our understanding of cholesterol metabolism.

Based on the RCT model, ApoA-I is an attractive target for therapeutic intervention. Experimental manipulations that increase production of ApoA-I have been associated with reduced atherogenicity. Human ApoA-I is protective in transgenic animal models (Shah et al. 1998; Rubin et al. 1991), and infusion of ApoA- $\mathrm{I}_{\text {Milano }}$ prevents atherosclerotic lesions and leads to regression of atherosclerotic plaques in human patients (below). Small-molecule compounds that increase the production of endogenous ApoA-I would be attractive therapeutic agents for treating dyslipidemias.

\section{Familial HDL Abnormalities}

As familial HDL-c deficiency is often associated with family histories of premature CAD, much effort has been directed to understanding the molecular defect(s) involved (Hovingh et al. 2005; von Eckardstein and Assmann, 1998; Calabresi and Franceschini, 1997). Increased HDL-c concentrations are generally accepted to be protective against the development of atherosclerosis and CAD, but studies have suggested that the underlying cause of the increased HDL-c may be important in whether it is protective.

The familial hypoalphalipoproteinemias are rare lipoprotein disorders characterized by low levels of plasma HDL. However, despite markedly reduced HDL levels, several of these conditions, including Tangier disease, fish eye disease, and LCAT deficiency, are not associated with premature atherosclerosis. However, some mutations in LCAT are known that do result in increased CAD (Vega and Grundy, 1996; Frohlich et al. 1990). Mutations leading to reduced CETP activity result in less CE being directed into Apo-B-containing particles (VLDL and LDL) and more remaining in HDL, resulting in increased HDL-c concentrations. Mutations leading to reduced hepatic lipase (HL) activity are rare and are associated with increased HDL-c concentrations and CAD.

\section{Tangier disease (TD)}

The ATP-binding cassette transporter A1 (ABCA1) protein regulates the efflux of cholesterol and phospholipids from the cell to apolipoprotein acceptors, 


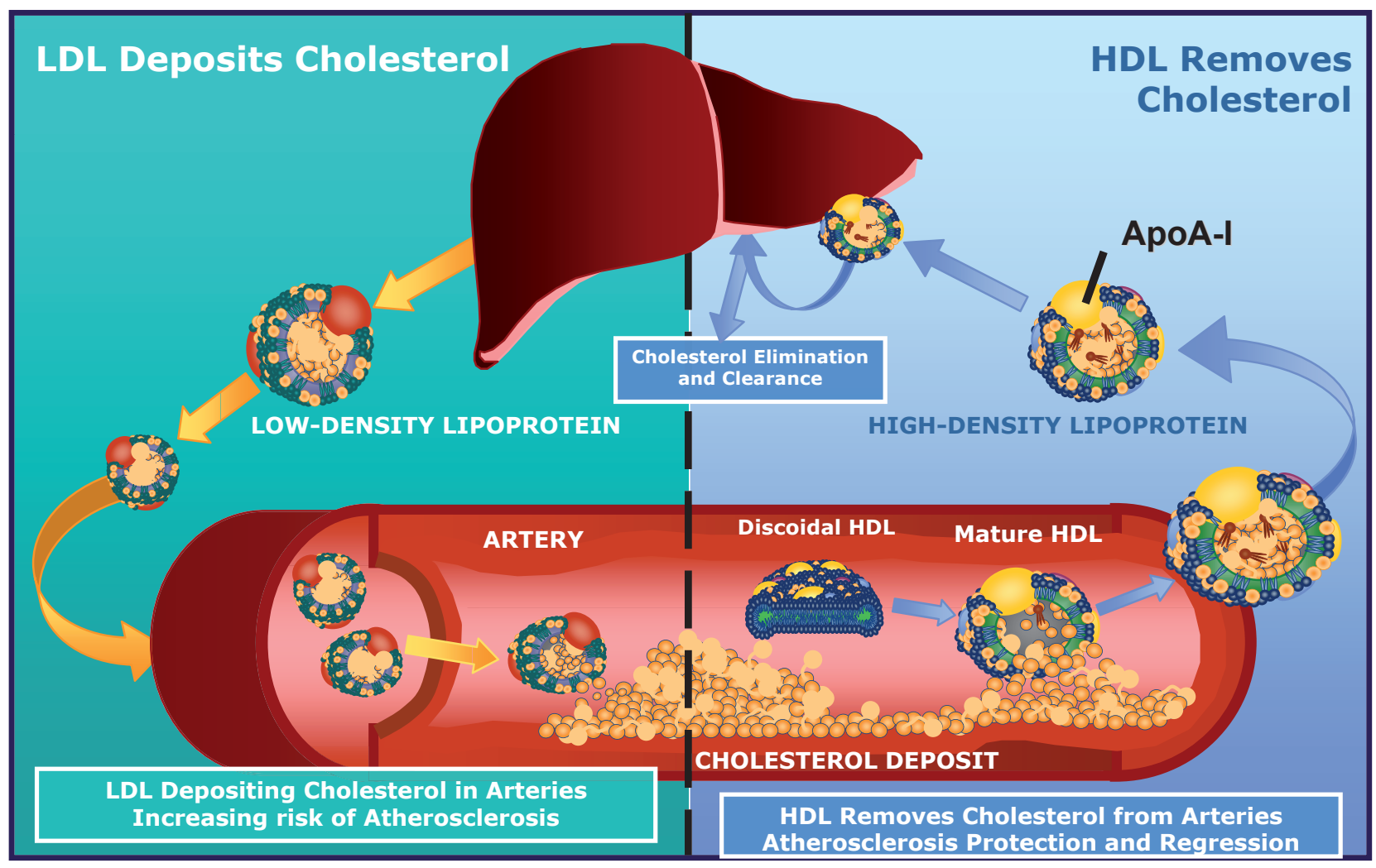

Figure 1.

the rate-limiting step in the removal of cellular cholesterol. Tangier disease is characterized by mutations in the ABCA1 gene, resulting in a defective ABCA1 protein and accumulation of cellular cholesterol, reduced plasma HDL-c, and increased risk for CAD; over 100 ABCA1 coding variants are known (Wang et al. 2000; Oram, 2000, 2001; Brunham et al. 2006; Kolovou et al. 2006; Tall and Wang, 2000).

The plasma of TD patients has essentially no ApoA-I-containing lipoprotein ( $\alpha$-LpA-I), which in normolipidemic plasma is the majority of HDL. Residual amounts of ApoA-I in TD plasma have electrophoretic pre $\beta 1-L p A-I$ mobility.

\section{CETP deficiency}

CETP deficiency causes hyperalphalipoproteinemia, that is, marked elevation of plasma HDL-c (e.g. Nagano et al. 2004; Yamashita et al. 2000). While the extent to which CETP deficiency is actually associated with protection against CAD is unclear, it gave rise to the idea of inhibiting CETP as a therapeutic strategy (Shah, 2007a; van der Steeg et al. 2005; Forrester et al. 2005; Doggrell, 2004; Barter and Kastelein, 2006; Barter et al. 2003).

\section{Lecithin: cholesterol acyltransferase} (LCAT) deficiency and fish eye disease LCAT esterifies free cholesterol in plasma and is important in the maturation of pre $\beta$-HDL (lipid-poor HDL) into $\alpha$-migrating HDL (spherical HDL). Primary (familial) LCAT deficiency (FLD) is a genetic disease associated with corneal opacity, anemia, and proteinuria with renal failure. It is caused by the complete or near absence of LCAT activity.

Fish eye disease (FED) is a related, milder condition in which there is some residual LCAT activity. Characteristic features of FED include corneal opacities, HDL-c below $10 \mathrm{mg} / \mathrm{dL}$, normal plasma cholesteryl esters, and elevated triglyceride levels. In FED, hypoalphalipoproteinemia is caused by marked hypercatabolism of ApoA-I and ApoA-Il.

\section{ApoA-I $\mathrm{I}_{\text {Milano }}$ and ApoA-I Paris}

ApoA- $\mathrm{I}_{\text {Milano }}$ and ApoA-I $\mathrm{I}_{\text {Paris }}$ are naturally occurring variants of ApoA-I associated with low rates of vascular disease and longevity in their carriers. Carriers of ApoA- $\mathrm{I}_{\text {Milano }}$ have reduced levels of LDL-c and very low levels of HDL. ApoA- $\mathrm{I}_{\text {Milano }}$ is characterized by a cysteine-for-arginine substitution at 
position 173 (R173C) of the protein sequence (Perez-Mendez et al. 2000; Klon et al. 2000). Despite this apparently pro-atherogenic profile, ApoA- $\mathrm{I}_{\text {Milano }}$ carriers appear to be at reduced risk for cardiovascular disease. In a pilot clinical study, a complex of recombinant ApoA-I $\mathrm{I}_{\text {Milano }}$ and 1-palmitoyl-2-oleoyl phosphatidylcholine (ETC-216) showed a significant reduction in coronary plaque burden after 5 weekly treatments, as assessed by intravascular ultrasound in patients with acute coronary syndrome (Nissen et al. 2003). ApoA- $\mathrm{I}_{\text {Paris }}$ is similarly characterized by a cysteinefor-arginine substitution, at position 151 (R151C).

These cysteine-for-arginine substitutions allow disulfide-linked dimers to form. The mutations appear to make the ApoA-I $\mathrm{I}_{\text {Milano }}$ and ApoA- $\mathrm{I}_{\text {Paris }}$ proteins functionally more effective than normal ApoA-I. In a rabbit model, HDL formulations of recombinant ApoA-I $\mathrm{I}_{\text {Milano-phospholipid complexes }}$ brought about rapid regression of a focal carotid atheroma and protection from myocardial infarction (Nicholls et al. 2005).

\section{HDL Infusion}

Several infusion studies with nascent HDL particles (ApoA-I/phospholipid complexes) have demonstrated the prevention and regression of atherosclerosis (Shah, 2007b). In cholesterol-fed rabbits, the effects of short-term administration of HDL and a statin on atherosclerosis were compared. Aortic atherosclerosis was established over 17 weeks in rabbits by balloon denudation and cholesterol feeding. Animals then received oral atorvastatin ( $5 \mathrm{mg} / \mathrm{kg}$ for 5 days) or two infusions of HDL particles ( $8 \mathrm{mg} / \mathrm{kg}$ ApoA-I) 2 days apart. Lesion size and composition were then assessed. HDL, but not atorvastatin, reduced lesion size by $36 \%$ (Nicholls et al. 2005).

In patients with heterozygous familial hypercholesterolemia, a single infusion with recombinant proApoA-I particles (precursor of ApoA-I) resulted in a 34\% sustained 10-day increase in cholesterol excretion, with a net removal of $5 \%-7 \%$ of total body cholesterol. Fecal sterol excretion was measured for 9 days before and 9 days after an intravenous infusion of the proApoA-I liposome complexes. Plasma ApoA-I and HDL-c levels increased transiently during the first $24 \mathrm{~h}$. Fecal excretion of cholesterol (neutral sterols and bile acids) increased in all subjects. Control infusions with only liposomes in two of the patients did not affect cholesterol excretion (Eriksson et al. 1999).
In patients with acute coronary syndrome, five weekly infusions of ApoA- $\mathrm{I}_{\text {Milano }}$ resulted in significant regression of coronary atherosclerosis (Nissen et al. 2003). This study evaluated the effect of intravenous recombinant ApoA- $\mathrm{I}_{\text {Milano-phospholipid }}$ complexes (ETC-216) on atheroma burden in patients with acute coronary syndromes $(n=47)$.

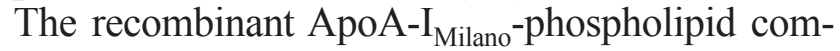
plex was administered intravenously, five doses at weekly intervals, and produced significant regression of coronary atherosclerosis, as assessed by intravascular ultrasound (IVUS). Remodeling of the arterial wall was shown to be a focal and heterogeneous process. After infusion of ETC-216, there was regression of coronary atherosclerosis, accompanied by reverse remodeling of the external elastic membrane, with no change in luminal dimensions. An average $11 \%$ reduction in atheroma volume resulted in the coronary segment containing the greatest atherosclerotic plaque (Nicholls et al. 2006).

In another study, the effects of reconstituted HDL on plaque burden, as assessed by IVUS, were examined. Sixty patients received 4 weekly infusions of placebo (saline), 111 received $40 \mathrm{mg} / \mathrm{kg}$ of reconstituted HDL (CSL-111), and 12 received CSL-111 at $80 \mathrm{mg} / \mathrm{kg}$. Changes in atheroma volume were assessed. The percentage change in atheroma volume was $-3.4 \%$ with CSL-111 and $-1.6 \%$ for placebo. Administration of the lower dose was associated with mild, self-limiting transaminase elevation, but was well tolerated. Short-term infusion of reconstituted HDL resulted in no significant reduction in percentage change in atheroma volume or nominal change in plaque volume compared with placebo, but did result in statistically significant improvement in the plaque characterization index and coronary score on quantitative coronary angiography (Tardif et al. 2007b).

Another line of research is the use of peptides that mimic ApoA-I. ApoA-I is a large protein, comprising 243 amino acids, making its recombinant preparation difficult and expensive. Additionally, intravenous administration is necessary, which is inconvenient. Efforts have been made at finding peptide mimetics that produce similar results to ApoA-I, but that would be easier to manufacture and administer (Pal and Pillarisetti, 2007).

\section{Methods to Increase HDL}

There is a continuing need for novel therapies that increase the biosynthesis of HDL, to inhibit the 
progression of and even bring about regression of atherosclerosis. The development of a small molecule drug that increases endogenous ApoA-I could provide a breakthrough therapy for treating cardiovascular disease, either as a stand-alone treatment or in combination. The parallels with ApoA-I infusion therapy are clear; while infusion therapies may be appropriate for short-term treatment, a small molecule approach could provide a long-term increase in ApoA-I, by chronic treatment. Targeting the patient's liver and small intestine to increase the transcription, synthesis, and secretion of ApoA-I is generally considered to be a promising approach to raising HDL-c levels (Rader, 2006, 2007a, 2007b; Toth, 2007; Nicholls and Nissen, 2007; but see Singh et al. 2007).

Much is now known about the regulatory sequences and transcription factors in the liver and small intestine that control ApoA-I transcription. The human gene encoding ApoA-I resides in an ApoA-I-CIII-AIV gene cluster, located at chromosome 11q23-q24 (Lai et al. 2005). The proximal ApoA-I promoter and enhancer contains multiple cis-acting regulatory elements to which various hepatocyte-specific and ubiquitous transcription factors bind to regulate liver-specific transcription, while expression in intestinal cells requires the ApoCIII promoter region (Walsh et al. 1993; Zannis et al. 2001).

Despite strong evidence connecting HDL to cardiovascular disease, there are currently no potent HDL elevators available. Existing methods of increasing HDL-c include life style changes, niacin, fibrates, estrogen, and to some degree, the statins. Life style modification can increase serum levels by $5 \%-15 \%$, while niacin, the drug most widely used to increase HDL-c, can increase it by $25 \%-35 \%$ at the highest doses, fibrates increase HDL-c by $10 \%-25 \%$, and statins by $5 \%-15 \%$ at moderate doses.

\section{Life style modification}

Much research suggests that the first step in increasing HDL-c levels should be life style modification. Regular aerobic exercise, loss of excess weight (fat), cessation of cigarette smoking, and changes in diet can all increase HDL-c levels (Ashen and Blumenthal, 2005; Kodama et al. 2007; Mooradian et al. 2006; Dullens et al. 2007). Dietary soy protein, soluble fiber, and plant sterol/ ester-containing margarines have all been shown to favorably increase the LDL:HDL ratio (Hermansen et al. 2003). Diets rich in wholegrain foods tend to increase serum HDL-c levels, while decreasing serum LDL-c, triacylglycerol levels, and blood pressure (Anderson, 2003). The effects of soy isoflavones are somewhat controversial (see Dullens et al. 2007).

Polyphenols have become a focus of research for their role in the prevention of cardiovascular disease and ability to raise HDL-c. Polyphenols are common components of the human diet, present in many foods and beverages of plant origin, including tea, wine, cocoa, and soy. Epidemiological studies have repeatedly shown an inverse association between the risk of myocardial infarction and the consumption of tea or wine and intake levels of some particular flavonoids, but no clear association has been found in clinical studies with primary clinical endpoints (Scalbert et al. 2005; Manach et al. 2005). In fact, in twelve intervention studies with differing polyphenol sources, six showed no effect on lipid parameters and six showed an improvement (Manach et al. 2005). Such inconclusive data has clouded the use of polyphenols (Zern and Fernandez, 2005).

Moderate consumption of red wine (one or two drinks per day) has consistently been associated with a reduced risk of cardiovascular diseases. This phenomenon has been used to explain the so-called "French Paradox," the relatively low rate of vascular disease in the French population, despite a high level of saturated fats in their diet (Poussier et al. 2005; Zern and Fernandez, 2005; Renaud and de Lorgeil, 1992; Burr, 1995; Schäfer et al. 2007).

The polyphenol resveratrol (trans-3,5,4'trihydroxystilbene), a stilbenoid antioxidant found in the skin of red grapes, wine, peanuts, and some berries, has been shown to exhibit alcoholindependent cardioprotective properties (Wang et al. 2005). Thus, mild-to-moderate alcohol consumption appears to be reasonable for many people; however, the potential risks associated with hepatic dysfunction and addiction may outweigh the benefits. Improvement in HDL-c with life style changes may often be sufficient; however, the interaction between genes and the environment may influence the magnitude of any improvement. Thus, when life style modifications are insufficient, medications are used.

Approaches to raise anti-atherogenic HDL-c have attracted much attention, because of expanding 
disease populations with low levels of HDL-c or high cholesterol, such as patients with type 2 diabetes, metabolic syndrome, dyslipidemia, and menopause. To date, several medications that have been approved that increase HDL-c and new approaches are being sought.

\section{Niacin}

Niacin is the most effective drug currently available to raise HDL-c levels. At higher pharmacological doses, niacin can reduce LDL-c, triglycerides, and apoB, while increasing HDL-c and ApoA-I (Chapman, 2005). These beneficial lipoprotein changes have been shown to translate into clinical benefits, decreasing morbidity and mortality from cardiovascular causes (McCormack and Keating, 2005). Nicotinic acid, as vitamin B3, is available without prescription; however, side effects tend to be frequent when taken under suboptimal conditions. Extended- or prolonged-release niacin (Niaspan), marketed by Kos Pharmaceuticals, decreases flushing, niacin's most common side effect and increased HDL-c by $\sim 25 \%$ from baseline at $200 \mathrm{mg} / \mathrm{kg}$ in clinical studies (Pal and Pillarisetti, 2007; McCormack and Keating, 2005; Birjmohun et al. 2004). With the discovery of the niacin receptor (HM74a, PUMA-G), several drug companies are working on developing novel agonists (Pal and Pillarisetti, 2007).

\section{Fibrates and PPAR agonists}

The peroxisome proliferator activated receptor (PPAR) nuclear receptors are believed to mediate the activity of the fibrates (fenofibrate activates PPAR $\alpha$ ) (Pal and Pillarisetti, 2007; Fruchart and Duriez, 2006). The fibrate class of drugs has been broadly used in the clinical treatment of dyslipidemia (Pal and Pillarisetti, 2007). The fibrates typically raise HDL levels by $10 \%-15 \%$ and can also lower triglyceride levels by up to $60 \%$, though they have little effect on LDL levels. In subjects with low HDL-c, gemfibrozil administration resulted in a $6 \%$ increase in HDL-c levels and a $22 \%$ decrease in CHD risk (Rubins et al. 1999).

The observed HDL-c elevation is the result of transcriptional induction of ApoA-I gene expression, mediated by the interaction of PPAR $\alpha$ with a functional PPRE, localized in the A site of the ApoA-I promoter (Vu-Dac et al. 1994). PPAR $\alpha$ regulates other genes involved in lipid metabolism, hemostasis, and inflammation, in response to fatty acids and fibrates, making it a candidate gene for risk of dyslipidemia, atherosclerosis, and coronary artery disease (Pal and Pillarisetti, 2007). Unfortunately, the development of effective medications in this drug class has been hampered by safety concerns and increased atherogenic lipids (Nissen et al. 2007a). For example, phase II results on Eli Lilly's PPAR $\alpha$ agonist, LY518674 demonstrated a dose-related increase in LDL-c that appeared to correlate with baseline triglycerides (Nissen et al. 2007a).

\section{Statins}

Statins are the most effective pharmacological means of lowering LDL-c. They inhibit 3-hydroxy3-methylglutaryl coenzyme A (HMG-CoA) reductase, the enzyme that catalyzes the ratelimiting step in cholesterol synthesis (Slater and MacDonald, 1988). By inhibiting cholesterol synthesis in the liver, statins activate hepatocyte LDL receptors and produce significant reductions in circulating LDL-c (Slater and MacDonald, 1988). Statins have been shown in many large, randomized clinical trials to reduce LDL-c, thereby reducing the risk of cardiovascular events and decreasing mortality (Nicholls et al. 2007).

Statins also increase levels of HDL-c and ApoA-I. In human HepG2 cells and hamster primary hepatocytes, statin treatment increased ApoA-I levels (Bonn et al. 2002). Furthermore, the statin response element has been shown to coincide with the PPAR $\alpha$ response element, known to confer fibrate responsiveness in the ApoA-I gene (Maejima et al. 2004). Statin therapy is associated with regression of coronary atherosclerosis, when LDL-c is substantially reduced and HDL-c is increased (Nicholls et al. 2007).

\section{Novel Drug Classes Under Investigation}

\section{Hormone replacement therapy}

Menopause is a pro-atherogenic state, associated with a significant increase in the incidence of coronary artery disease (Schnatz and Schnatz, 2006). Estrogen replacement therapies, such as conjugated equine estrogen (CEE, Premarin), reduce the risk of CAD in postmenopausal women (Lamon-Fava et al. 2006). Hormone replacement therapy (HRT) is associated with increased ApoA-I and HDL and 
decreased LDL and total cholesterol levels. ApoA-I levels were increased most by use of estrogen alone (Lamon-Fava et al. 2006).

\section{Rimonabant}

Rimonabant, a cannabinoid-1 receptor blocker, is being investigated for use in reducing body weight and improving cardiometabolic risk factors in overweight or obese patients (Forrester and Shah, 2006). In one randomized, controlled trial, the mean weight loss was 19 pounds at 2 years. In the high-dose group, $39 \%$ lost $10 \%$ of their initial body weight, compared with $15 \%$ in the low-dose cohort and $12 \%$ of those receiving a placebo. The number of patients with metabolic syndrome at baseline was reduced by about $50 \%$ with rimonabant $20 \mathrm{mg}$. Concomitant with the weight loss, the patients exhibited an $11 \%$ decrease in triglyceride and a 27\% increase in HDL cholesterol (Forrester and Shah, 2006). This drug is currently approved in many markets under the brand name Acomplia. In June 2007, the U.S. FDA's Endocrinologic and Metabolic Drugs Advisory Committee did not recommend approval of rimonabant for weight management.

\section{CETP inhibitors}

Cholesterol ester transfer protein (CETP) is a glycoprotein that facilitates the transfer of cholesteryl esters from HDL-c to Apo B-containing lipoproteins in exchange for triglycerides (Pal and Pillarisetti, 2007). Subjects with CETP deficiencies, because of CETP gene defects, have elevated plasma levels of HDL-c and ApoA-I. However the possibly anti-atherogenic role of CETP is complex because, despite raising HDL-c levels, it also slows the metabolism of HDL-c (Rader, 2006, 2007a). To date, two CETP inhibitors, JTT-705 and torcetrapib, have been evaluated clinically and have shown efficacy, in that they do increase HDL-c (Rader, 2006, 2007a; Barter et al. 2007).

In early clinical studies, torcetrapib showed a pronounced effect on plasma lipoproteins in patients with low HDL-c levels, and reduced the levels of LDL-c and apolipoprotein B, both as a monotherapy and in combination with atorvastatin (Rader, 2006, 2007a). After the phase II dose-ranging trials, torcetrapib, despite being associated with a substantial increase in HDL-c and decrease in LDL cholesterol, was also associated with an increase in blood pressure, and no decrease in the progression of coronary atherosclerosis (Nissen et al. 2007b). The lack of efficacy may be related to the mechanism of action of this drug class or to molecule-specific adverse effects (Rader, 2007b; Barter et al. 2007). After demonstrating efficacy in phase II, no additional data has yet been published on JTT-705.

\section{AGI-1067}

Inflammation has a fundamental role in mediating all stages of atherosclerotic disease. AGI-1067 (probucol monosuccinate, succinobucol) is being assessed for the treatment of restenosis and possibly atherosclerosis. The pharmacological activities of AGI-1067 are the ability to block the expression of oxidation-sensitive inflammatory genes, including genes that encode vascular cell adhesion molecule-1 and monocyte chemotactic protein-1. In a placebo-controlled, randomized study of the effects of AGI-1067 on coronary atherosclerosis, some regression was observed in patients treated with AGI-1067, but it was not significantly different from placebo. Moreover, HDL-c was reduced by $14 \%$ with AGI-1067 treatment and 1\% with placebo (Tardif et al. 2007a).

\section{Phospholipids}

Phosphatidylinositol can stimulate reverse cholesterol transport by enhancing the flux of cholesterol into HDL and by promoting the transport of HDL-c to the liver and bile. Currently, phosphatidylinositol (PI) is being evaluated in human subjects. At doses as high as $5.6 \mathrm{~g} /$ day, PI demonstrated increases of HDL-c of $18 \%$ over a 2 -week period, and a decrease in triglycerides by $36 \%$ in fed subjects. This product is in clinical development (Burgess et al. 2005).

CSL-111 is a reconstituted HDL, consisting of ApoA-I from human plasma combined with soybean phosphatidylcholine, that resembles native HDL (Tardiff et al. 2007b). In a 183-patient phase II clinical trial, there was no significant reduction in atheroma or plaque volume, compared with the placebo, but there was a statistically significant improvement in the plaque characterization index and coronary score on quantitative coronary angiography (Tardiff et al. 2007b).

\section{Conclusions}

The lipid hypothesis, now more than 100 years old, is based on the idea that dyslipidemia is central to 
atherosclerosis. Its validity was ultimately demonstrated by major epidemiological studies, demonstrating that cholesterol levels were linearly related to coronary heart disease mortality.

Further epidemiological studies showed an inverse relationship between blood levels of HDL-c and the incidence of clinically significant atherosclerosis; it appears that each $1 \mathrm{mg} / \mathrm{dL}$ increment in serum HDL-c is associated with a $2 \%-3 \%$ decrement in cardiovascular risk (Boden, 2000). While our understanding of the mechanism(s) by which HDL alters atherosclerotic disease remain(s) incomplete, it is believed that elevated levels of HDL increase the transport of cholesterol from arteries and tissues to the liver for excretion.

Based on the RCT model (Fig. 1), ApoA-I is an attractive target for therapeutic intervention. Experimental manipulations to increase ApoA-I have been shown to reduce atherogenicity. Human ApoA-I is protective in transgenic animal models (Shah et al. 1998; Rubin et al. 1991) and infusion of ApoA-I $\mathrm{I}_{\text {Milano }}$ prevents atherosclerotic lesions and leads to regression of atherosclerotic plaques in human patients.

There is an ongoing need for novel therapies to increase the biosynthesis of HDL, to inhibit the progression of and even bring about regression of atherosclerosis. The development of a small molecule drug that increased endogenous ApoA-I would be a major advance in treating lipid-related cardiovascular disease.

Targeting the patient's liver and small intestine to increase the transcription, synthesis, and secretion of ApoA-I is generally considered a promising approach for raising HDL-c levels. Currently, our ability to raise HDL is limited; life style, including dietary, changes, niacin, fibrates, estrogen, and to some degree, the statins do raise HDL, but not very potently. Several new drug classes are under investigation in this very active field.

\section{References}

Anderson, J.W. 2003. Whole grains protect against atherosclerotic cardiovascular disease. Proc. Nutr. Soc., 62:135-42.

Ashen, M.D. and Blumenthal, R.S. 2005. Clinical practice. Low HDL cholesterol levels. N. Engl. J. Med., 353:1252-60.

Assmann, G., Funke, H. and Schriewer, H. 1982. The relationship of HDL-apolipoprotein A-I and HDL-Cholesterol to risk factors of coronary heart disease: initial results of the prospective epidemiological study in company employees in Westfalia. J. Clin. Chem. Clin. Biochem., 20:287-9.

Barter, P.J., Brewer, H.B., Jr, Chapman, M.J. et al. 2003. Cholesteryl ester transfer protein: a novel target for raising HDL and inhibiting atherosclerosis. Arterioscler. Thromb. Vasc. Biol., 23:160-7.
Barter, P.J., Caulfield, M., Eriksson, M. et al. 2007. Effects of torcetrapib in patients at high risk for coronary events. New Engl. J. Med., 357:2109-22.

Barter, P.J. and Kastelein, J.J. 2006. Targeting cholesteryl ester transfer protein for the prevention and management of cardiovascular disease. J. Am. Coll. Cardiol., 47:492-9.

Birjmohun, R.S., Hutten, B.A., Kastelein, J.J. et al. 2004. Increasing HDL cholesterol with extended-release nicotinic acid: from promise to practice. Neth. J. Med., 62:229-34.

Boden, W.E. 2000. High-density lipoprotein cholesterol as an independent risk factor in cardiovascular disease: assessing the data from Framingham to the Veterans Affairs High-Density Lipoprotein Intervention Trial. Am. J. Cardiol., 86:19L-22L.

Bonn, V., Cheung, R.C., Chen, B. et al. 2002. Simvastatin, an HMG-CoA reductase inhibitor, induces the synthesis and secretion of apolipoprotein $\mathrm{AI}$ in HepG2 cells and primary hamster hepatocytes. Atherosclerosis, 163:59-68.

Brunham, L.R., Singaraja, R.R. and Hayden, M.R. 2006. Variations on a gene: rare and common variants in ABCA1 and their impact on HDL cholesterol levels and atherosclerosis. Annu. Rev. Nutr., 26:105-29.

Burgess, J.W., Neville, T.A., Rouillard, P. et al. 2005. Phosphatidylinositol increases HDL-C levels in humans. J. Lipid Res., 46:350-5.

Burr, M.L. 1995. Explaining the French paradox. J. R. Soc. Health, 115:217-9.

Calabresi, L. and Franceschini, G. 1997. High density lipoprotein and coronary heart disease: insights from mutations leading to low high density lipoprotein. Curr. Opin. Lipidol., 8:219-24.

Chapman, M.J. 2005. Beyond the statins: new therapeutic perspectives in cardiovascular disease prevention. Cardiovasc. Drugs Ther., 19:135-9.

Doggrell, S.A. 2004. Raising high-density lipoprotein cholesterol with inhibitors of cholesteryl ester transfer protein - a new approach to coronary artery disease. Expert Opin. Investig. Drugs, 13:1365-8.

Downs, J.R., Clearfield, M., Weis, S. et al. 1998. Primary prevention of acute coronary events with lovastatin in men and women with average cholesterol levels: results of AFCAPS/TexCAPS. Air Force/Texas Coronary Atherosclerosis Prevention Study. JAMA, 279:1615-22.

Eriksson, M., Carlson, L.A., Miettinen, T.A. et al. 1999. Stimulation of fecal steroid excretion after infusion of recombinant proapolipoprotein A-I. Potential reverse cholesterol transport in humans. Circulation, 100:594-8.

Frohlich, J., Westerlund, J., Sparks, D. et al. 1990. Familial hypoalphalipoproteinemias. Clin. Invest. Med., 13:202-10.

Forrester, J.S., Makkar, R. and Shah, P.K. 2005. Increasing high-density lipoprotein cholesterol in dyslipidemia by cholesteryl ester transfer protein inhibition: an update for clinicians. Circulation, 111:1847-54

Forrester, J.S. and Shah, P.K. 2006. Emerging strategies for increasing high-density lipoprotein. Am. J. Cardiol., 98:1542-9.

Fruchart, J.C. and Duriez, P. 2006. Mode of action of fibrates in the regulation of triglyceride and HDL-cholesterol metabolism. Drugs Today, 42:39-64

Gordon, T., Castelli, W.P., Hjortland, M.C. et al. 1977. High density lipoprotein as a protective factor against coronary heart disease. The Framingham Study. Am. J. Med., 62:707-14.

Hermansen, K., Dinesen, B., Hoie, L.H. et al. 2003. Effects of soy and other natural products on LDL:HDL ratio and other lipid parameters: a literature review. Adv. There, 20:50-78.

Hovingh, G.K., de Groot, E., van der Steeg, W. et al. 2005. Inherited disorders of HDL metabolism and atherosclerosis. Curr. Opin. Lipidol., 16:139-45.

Klon, A.E., Jones, M.K., Segrest, J.P. et al. 2000. Molecular belt models for the apolipoprotein A-I Paris and Milano mutations. Biophys. J., 79:1679-85.

Kodama, S., Tanaka, S., Saito, K. et al. 2007. Effect of aerobic exercise training on serum levels of high-density lipoprotein cholesterol: a meta-analysis. Arch. Intern. Med., 167:999-1008. 
Kolovou, G.D., Mikhailidis, D.P., Anagnostopoulou, K.K. et al. 2006 Tangier disease four decades of research: a reflection of the importance of HDL. Curr. Med. Chem., 13:771-82.

Lai, C.Q., Parnell, L.D. and Ordovas, J.M. 2005. The APOA1/C3/A4/A5 gene cluster, lipid metabolism and cardiovascular disease risk. Curr. Opin. Lipidol., 16:153-66.

Lamon-Fava, S., Postfai, B., Diffenderfer, M. et al. 2006. Role of the estrogen and progestin in hormonal replacement therapy on apolipoprotein A-I kinetics in postmenopausal women. Arterioscler. Thromb. Vasc. Biol., 26:385-91.

McCormack, P.L. and Keating, G.M. 2005. Prolonged-release nicotinic acid: a review of its use in the treatment of dyslipidaemia. Drugs, 65:2719-40.

Maejima, T., Yamazaki, H., Aoki, T. et al. 2004. Effect of pitavastatin on apolipoprotein A-I production in HepG2 cells. Biochem. Biophys. Res. Commun., 324:835-9.

Manach, C., Mazur, A. and Scalbert, A. 2005. Polyphenols and prevention of cardiovascular diseases. Curr. Opin. Lipidol., 16:77-84.

Mooradian, A.D., Haas, M.J. and Wong, N.C. 2006. The effect of select nutrients on serum high-density lipoprotein cholesterol and apolipoprotein A-I levels. Endocr. Rev., 27:2-16.

Nagano, M., Yamashita, S., Hirano, K. et al. 2004. Molecular mechanisms of cholesteryl ester transfer protein deficiency in the Japanese. J. Atheroscler. Thromb., 11:110-21.

Nicholls, S.J. and Nissen, S.E. 2007. New targets of high-density lipoprotein therapy. Curr. Opin. Lipidol., 18:421-6.

Nicholls, S.J., Cutri, B., Worthley, S.G. et al. 2005. Impact of short-term administration of high-density lipoproteins and atorvastatin on atherosclerosis in rabbits. Arterioscler. Thromb. Vasc. Biol., $25: 2416-21$

Nicholls, S.J., Tuzcu, E.M., Sipahi, I. et al. 2006. Relationship between atheroma regression and change in lumen size after infusion of apolipoprotein A-I Milano. J. Am. Coll. Cardiol., 47:992-7.

Nicholls, S.J., Tuzcu, E.M., Sipahi, I. et al. 2007. Statins, high-density lipoprotein cholesterol, and regression of coronary atherosclerosis. JAMA, 297:499-508.

Nissen, S.E., Nicholls, S.J., Wolski, K. et al. 2007a. Effects of a potent and selective PPAR-alpha agonist in patients with atherogenic dyslipidemia or hypercholesterolemia: two randomized controlled trials. JAMA, 297:1362-73.

Nissen, S.E., Tardif, J.C., Nicholls, S.J. et al. 2007b. Effect of torcetrapib on the progression of coronary atherosclerosis. N. Engl. J. Med., 356:1304-16.

Nissen, S.E., Tsunoda, T., Tuzcu, E.M. et al. 2003. Effect of recombinant ApoA-I Milano on coronary atherosclerosis in patients with acute coronary syndromes: a randomized controlled trial. JAMA, 290:2292-300.

Oram, J.F. 2000. Tangier disease and ABCA1. Biochim. Biophys. Acta., 1529:321-30.

Oram, J.F. 2001. Novel approaches to treating cardiovascular disease: lessons from Tangier disease. Expert Opin. Investig. Drugs, 10:427-38.

Pal, M. and Pillarisetti, S. 2007. HDL elevators and mimetics-emerging therapies for atherosclerosis. Cardiovasc. Hematol. Agents Med. Chem., 5:55-66.

Perez-Mendez, O., Bruckert, E., Franceschini, G. et al. 2000. Metabolism of apolipoproteins AI and AII in subjects carrying similar apoAI mutations, apoAI Milano and apoAI Paris. Atherosclerosis, $148: 317-25$

Poussier, B., Cordova, A.C., Becquemin, J.P. et al. 2005. Resveratrol inhibits vascular smooth muscle cell proliferation and induces apoptosis. J. Vasc. Surg., 42:1190-7.

Rader, D.J. 2006. Molecular regulation of HDL metabolism and function: implications for novel therapies. J. Clin. Invest., 116:3090-100.

Rader, D.J. 2007a. Mechanisms of disease: HDL metabolism as a target for novel therapies. Nat. Clin. Pract. Cardiovasc. Med., 4:102-9.

Rader, D.J. 2007b. Illuminating HDL-Is it still a viable therapeutic target? New Engl. J. Med., 357:2180-83.
Renaud, S. and de Lorgeril, M. 1992. Wine, alcohol, platelets, and the French paradox for coronary heart disease. Lancet, 339:1523-6.

Robins, S.J., Collins, D., Wittes, J.T. et al. 2001. Veterans Affairs HighDensity Lipoprotein Intervention Trial. Relation of gemfibrozil treatment and lipid levels with major coronary events: VA-HIT: a randomized controlled trial. JAMA, 285:1585-91.

Rubin, E.M., Krauss, R.M., Spangler, E.A. et al. 1991. Inhibition of early atherogenesis in transgenic mice by human apolipoprotein AI. Nature, 353:265-7.

Rubins, H.B., Robins, S.J., Collins, D. et al. 1999. Gemfibrozil for the secondary prevention of coronary heart disease in men with low levels of high-density lipoprotein cholesterol. Veterans Affairs HighDensity Lipoprotein Cholesterol Intervention Trial Study Group. N. Engl. J. Med., 341:410-8.

Scalbert, A., Manach, C., Morand, C. et al. 2005. Dietary polyphenols and the prevention of diseases. Crit. Rev. Food Sci. Nutr., 45:287-306.

Schaefer, E.J., Eisenberg, S. and Levy, R.I. 1978. Lipoprotein apoprotein metabolism. J. Lipid Res., 19:667-87.

Schäfer, C., Parlesak, A., Eckoldt, J. et al. 2007. Beyond HDL-cholesterol increase: phospholipid enrichment and shift from HDL3 to HDL2 in alcohol consumers. J. Lipid Res., 48:1550-8.

Schnatz, P.F. and Schnatz, J.D. 2006. Dyslipidemia in menopause: mechanisms and management. Obstet. Gynecol. Surv., 61:608-13.

Schultz, J.R., Verstuyft, J.G., Gong, E.L. et al. 1993. Protein composition determines the anti-atherogenic properties of HDL in transgenic mice. Nature, 365:762-4.

Shah, P.K. 2007a. Inhibition of CETP as a novel therapeutic strategy for reducing the risk of atherosclerotic disease. Eur. Heart J., 28:5-12.

Shah, P.K. 2007b. Apolipoprotein A-I/HDL infusion therapy for plaque stabilization-regression: a novel therapeutic approach. Curr. Pharm. Des., 13:1031-8.

Shah, P.K., Nilsson, J., Kaul, S. et al. 1998. Effects of recombinant apolipoprotein A-I(Milano) on aortic atherosclerosis in apolipoprotein E-deficient mice. Circulation, 97:780-5.

Singh, I.M., Shishehbor, M.H. and Ansell, B.J. 2007. High-density lipoprotein as a therapeutic target: a systematic review. JAMA, 298:786-98.

Slater, E.E. and MacDonald, J.S. 1988. Mechanism of action and biological profile of HMG CoA reductase inhibitors. A new therapeutic alternative. Drugs, 36(suppl 3):72-82.

Steinberg, D. 2004. Thematic review series: the pathogenesis of atherosclerosis. An interpretive history of the cholesterol controversy: part I. J. Lipid Res., 45:1583-93.

Steinberg, D. 2005a. Thematic review series: the pathogenesis of atherosclerosis. An interpretive history of the cholesterol controversy: part II: the early evidence linking hypercholesterolemia to coronary disease in humans. J. Lipid Res., 46:179-90.

Steinberg, D. 2005b. Thematic review series: the pathogenesis of atherosclerosis: an interpretive history of the cholesterol controversy, part III: mechanistically defining the role of hyperlipidemia. J. Lipid Res., 46:2037-51

Steinberg, D. 2006a. The pathogenesis of atherosclerosis. An interpretive history of the cholesterol controversy, part IV: the 1984 coronary primary prevention trial ends it—almost. J. Lipid Res., 47:1-14.

Steinberg, D. 2006b. Thematic review series: the pathogenesis of atherosclerosis. An interpretive history of the cholesterol controversy, part V: the discovery of the statins and the end of the controversy. J. Lipid Res., 47:1339-51.

Tall, A.R. and Wang, N. 2000. Tangier disease as a test of the reverse cholesterol transport hypothesis. J. Clin. Invest., 106:1205-7.

Tardif, J.C., Grégoire, J., L'Allier, P.L. et al. 2007a. Effects of the antioxidant succinobucol (AGI-1067) on human atherosclerosis in a randomized clinical trial. Atherosclerosis, in press.

Tardif, J.C., Grégoire, J., L'Allier, P.L. et al. 2007b. Effect of rHDL on Atherosclerosis-Safety and Efficacy (ERASE) Investigators. Effects of reconstituted high-density lipoprotein infusions on coronary atherosclerosis: a randomized controlled trial. JAMA, 297:1675-82. 
Toth, P.P. 2007. Reducing cardiovascular risk by targeting high-density lipoprotein cholesterol. Curr. Atheroscler. Rep., 9:81-8.

van der Steeg, W.A., El-Harchaoui, K., Kuivenhoven, J.A. et al. 2005. Increasing high-density lipoprotein cholesterol through cholesteryl Ester transfer protein inhibition: a next step in the fight against cardiovascular disease? Curr. Drug Targets Cardiovasc. Haematol. Disord., 5:481-8.

Vega, G.L. and Grundy, S.M. 1996. Hypoalphalipoproteinemia (low high density lipoprotein) as a risk factor for coronary heart disease. Curr. Opin. Lipidol., 7:209-16.

Verschuren, W.M., Jacobs, D.R., Bloemberg, B.P. et al. 1995. Serum total cholesterol and long-term coronary heart disease mortality in different cultures. Twenty-five-year follow-up of the seven countries study. JAMA, 274:131-6.

von Eckardstein, A. and Assmann, G. 1998. High density lipoproteins and reverse cholesterol transport: lessons from mutations. Atherosclerosis, 137(suppl):S7-11.

Vu-Dac, N., Schoonjans, K., Laine, B. et al. 1994. Negative regulation of the human apolipoprotein A-I promoter by fibrates can be attenuated by the interaction of the peroxisome proliferator-activated receptor with its response element. J. Biol. Chem., 269:31012-8.

Walldius, G., Jungner, I., Holme, I. et al. 2001. High apolipoprotein B., low apolipoprotein A-I, and improvement in the prediction of fatal myocardial infarction (AMORIS study): a prospective study. Lancet, 358:2026-33.
Walsh, A., Azrolan, N., Wang, K. et al. 1993. Intestinal expression of the human apoA-I gene in transgenic mice is controlled by a DNA region 3 ' to the gene in the promoter of the adjacent convergently transcribed apoC-III gene. J. Lipid Res., 34:617-23.

Wang, J., Burnett, J.R., Near, S. et al. 2000. Common and rare ABCA1 variants affecting plasma HDL cholesterol. Arterioscler. Thromb. Vasc. Biol., 20:1983-9.

Wang, Z., Zou, J., Cao, K. et al. 2005. Dealcoholized red wine containing known amounts of resveratrol suppresses atherosclerosis in hypercholesterolemic rabbits without affecting plasma lipid levels. Int. $J$. Mol. Med., 16:533-40.

Yamashita, S., Maruyama, T., Hirano, K. et al. 2000. Molecular mechanisms, lipoprotein abnormalities and atherogenicity of hyperalphalipoproteinemia. Atherosclerosis, 152:271-85.

Yusuf, S., Hawken, S., Ounpuu, S. et al. 2004. Effect of potentially modifiable risk factors associated with myocardial infarction in 52 countries (the INTERHEART study): case-control study. Lancet, 364:937-52.

Zannis, V.I., Kan, H.Y., Kritis, A. et al. 2001. Transcriptional regulatory mechanisms of the human apolipoprotein genes in vitro and in vivo. Curr. Opin. Lipidol., 12:181-207.

Zern, T.L. and Fernandez, M.L. 2005. Cardioprotective effects of dietary polyphenols. J. Nutr., 135:2291-4. 